\title{
KATARZYNA THIEL-JAŃCZUK
}

Université Adam Mickiewicz

\section{L'auteur face au « spectacle » : vers une filiation non narrative}

e concept de "récit de filiation » a été forgé par " pratique narrative vis[ant] la saisie de soi par le biais de I'héritage et dans un rapport de descendance à l'autre ${ }^{1}$, pratique qui s'est développée au sein des écritures autobiographique et autofictionnelle contemporaines. Reconnu par Viart lui-même en tant que correspondant littéraire de la pensée de l'Autre dans les sciences humaines², le récit de filiation a également été emblématique d'un " retour du sujet » proclamé depuis les années 1980, qui comprenait la capacité à narrer propre à ce sujet comme l'un des principaux moyens de constitution de son identité. Toutefois, vingt ans après et dans de nouvelles circonstances médiatiques, il semble intéressant de poser les questions suivantes : que devient la filiation littéraire à l'époque des médias visuels ? Est-il toujours possible de l'envisager uniquement en termes narratifs ? Sans prétendre apporter une réponse exhaustive à ces questions, j'essaierai de signaler quelques aspects d'une possible filiation non narrative, en prenant comme point de départ une thèse

${ }^{1} \mathrm{M}$. Prosowska, Le récit de filiation dans la littérature contemporaine en France et en Pologne, thèse de doctorat non publiée, sous la direction de J. Lis, Poznań, Université Adam Mickiewicz, 2007, p. 7.

2 D. Viart, "Filiations littéraires ", [dans :] J. Baetens, D. Viart (dir.), Écritures contemporaines 2. États du roman contemporain, Actes du colloque de Calaceite, 6-13 juillet 1996, Paris-Caen, Lettres Modernes Minard, 1999, p. 123. 
récente de Vincent Kaufmann sur la "spectacularisation de l'auteur ${ }^{3}$ qui se réfère à la question s'imposant de plus en plus depuis une dizaine d'années, à savoir la visibilité médiatique de ce dernier. Je me référerai aussi, pour illustrer mon propos, à l'exemple de la filiation non narrative qui unit Marie Modiano, chanteuse et écrivaine française, et son père, l'écrivain Patrick Modiano (prix Nobel 2014).

\section{La filiation narrative et "la culture de l'écrit »}

Aussi bien pour Dominique Viart que pour Laurent Demanze, qui a proposé en 2008 une étude plus systématique du récit de filiation chez trois écrivains français contemporains ${ }^{4}$, la transmission d'un héritage - littéraire, artistique ou biologique - qui se trouve au cœur de ce concept, s'effectue par le biais de la narration. Ainsi, pour Demanze, "l'écrivain ne saurait se saisir sans l'élan narratif d'un récit qui organise et interprète le sens incertain d'une vie " ${ }^{5}$. De même, pour Viart, "le récit de filiation, qu'il prenne des formes autobiographiques ou fictives, est [...] le mode privilégié de l'écriture du sujet ${ }^{6}$. Tout en considérant donc la narration comme la seule réalité, le récit de filiation se soucie moins de la référence que de la valorisation de l'altérité : "Si l'écrivain contemporain s'approprie des lignes de vie de son ascendance [...], il n'en demeure pas moins qu'il se saisit comme autre ${ }^{7}-$

\footnotetext{
${ }^{3}$ V. Kaufmann, Les dernières nouvelles du spectacle. Ce que les médias font à la littérature, Paris, Seuil, 2017 (ebook). Ce terme renvoie bien sûr à l'idée du " spectacle » de Guy Debord, développée dans La société du spectacle (1967) qui est une critique de la société de consommation où le « rapport social entre des personnes [est] médiatisé par des images » (G. Debord, La société du spectacle, 3e édition, Paris, Gallimard 1992, p. 10). ${ }^{4}$ Cf. L. Demanze, Encres orphelines. Pierre Bergounioux, Gérard Macé, Pierre Michon, Paris, José Corti, 2008.

5 Ibidem, p. 373.

${ }^{6}$ D. Viart, "Filiations littéraires », op. cit., p. 124.

${ }^{7}$ L. Demanze, Encres orphelines. Pierre Bergounioux, Gérard Macé, Pierre Michon, op. cit., p. 372.
} 
constate Demanze tandis que Viart affirme : "Le sujet de notre temps [...] ne peut se connaître que par le détour d'autrui ${ }^{8}{ }^{8}$. Ces propos renouent, certes, avec des approches narratives du sujet en sciences humaines proposées par un Paul Ricœur ou une Julia Kristeva qui sont volontiers cités par les deux théoriciens. Mais ils permettent aussi de comprendre le récit de filiation, en particulier lorsqu'il concerne un écrivain, en tant que forme mineure de retour à ce que Kaufmann appelle, dans une perspective médiologique développée par Régis Debray, " la culture du livre et de l'écrit ${ }^{9}$. Cette culture, organisée autour de la figure du " grand écrivain " et de sa "panthéonisation ${ }^{10}$, et dont les derniers avatars sont "la mort de l'auteur " et " la textualité ${ }^{11}$, a été ébranlée à partir des années 1970 par les cultures audiovisuelle et numérique qui imposent de nouvelles formes de légitimation de l'écrivain. Olivier Nora dans une étude consacrée à la "visite au grand-écrivain ", qui constituait autant un rituel de consécration qu'un rituel de transmission d'un patrimoine littéraire en particulier au temps de la " Troisième République des lettres ${ }^{12}$, observe qu'à l'époque des médias audiovisuels "l'effet charismatique propre à l'écriture ne repose plus sur la lecture, mais sur l'audition et sur la vision ${ }^{13}$. II n'est donc plus question de la (re)connaissance de l'œuvre, mais de celle de la présence médiatique de l'écrivain, voire de "l'économie de l'attention ${ }^{14}$ quantifiable non plus en nombre de lecteurs, mais d'auditeurs et de spectateurs, sinon d'entrées sur les pa-

${ }^{8}$ D. Viart, "Filiations littéraires ", op. cit., p. 123.

${ }^{9} \mathrm{~V}$. Kaufmann, Les dernières nouvelles du spectacle, op. cit.

${ }^{10} \mathrm{Cf}$. J.-C. Bonnet, Naissance du Panthéon. Essai sur le culte des grands hommes, Paris, Fayard, 1998.

$11 \mathrm{~V}$. Kaufmann, Les dernières nouvelles du spectacle, op. cit.

12 Cf. A. Compagnon, La Troisième République des lettres. De Flaubert à Proust, Paris, Seuil, 1983.

13 O. Nora, "La visite au grand écrivain ", [dans :] P. Nora (dir.), Les lieux de mémoire, Paris, Gallimard, 1986, t. 2 (« La Nation »), vol. 3, p. 582.

${ }^{14} \mathrm{~V}$. Kaufmann, Les dernières nouvelles du spectacle, op. cit. 
ges fan Facebook : les écrivains " sont de moins en moins lus et de plus en plus vus ", constate la chercheuse espagnole Pilar Andrade Boué en se fondant sur un exemple significatif : la ministre de la Culture du gouvernement de François Hollande, Fleur Pellerin, lors d'un entretien après le déjeuner avec Patrick Modiano en octobre 2014, a avoué n'avoir lu aucun de ses livres ${ }^{15}$.

Selon Kaufmann, la voie vers cette " économie de l'attention " a été ouverte par un essor considérable de l'écriture autobiographique depuis les années 1980. C'est elle qui a contribué à démocratiser l'écriture, à abolir d'anciens rapports de hiérarchie symbolique aussi bien entre l'écrivain et le lecteur qu'entre le grand-écrivain et un écrivainadepte. En revanche, pour Henri Raczymow qui, dans La mort du grand écrivain. Essai sur la fin de la littérature réfléchit, avant Kaufmann, sur l'influence des médias sur la littérature, c'est l'émission télévisée Apostrophes de Bernard Pivot qui a marqué une étape décisive dans la mise en place de l'économie de l'attention en perturbant les mécanismes de transmission de l'héritage littéraire : " Dans l'ère aujourd'hui révolue des années 1980 ", note l'auteur de La mémoire trouée, " où un seul homme, Bernard Pivot, régnait sur l'édition française, comme seule instance de légitimation, le téléspectateur, vous et moi, se disait : ces auteurs sont réunis sur le plateau d'Apostrophes, il s'agit donc d'écrivains. Sous-entendu : ceux qui n'y paraissent pas, ne le sont pas vraiment, ou le sont moins que les autres [...] ${ }^{16}$. Dans la culture audiovisuelle, semble suggérer Raczymow, la transmission est impossible, car au lieu de "tuer le père ", qui est, dans la culture de l'écrit, un geste d'institution symbolique du jeune écrivain voulant prendre la place de son prédécesseur (tel le geste

15 P. A. Boué, " Jean-Marie Gustave Le Clézio et Patrick Modiano dans le champ intellectuel européen ", [dans :] Carnets [Online], Deuxième série, 2017, n० 9, http://journals.openedition.org/carnets/2052.

${ }^{16} \mathrm{H}$. Raczymow, La mort du grand écrivain. L'essai sur la fin de la littérature, Paris, Stock, 1996 (ebook). 
provocateur de la " miction " par Sartre du tombeau de Chateaubriand), " on s' [y] acharne sur un cadavre, devenu indifférent à l'opinion publique captive de la seule télévision ${ }^{17}$.

Pourtant, même si l'économie de l'attention a pour conséquence la banalisation du livre, voire même celle du " fait littéraire » (J. Bersani) en général, il paraît difficile aujourd'hui de passer outre l'image qu'un écrivain construit autour de sa personne. Pour Pilar Andrade Boué, la visibilité de l'écrivain est même, à l'heure actuelle, indissociable de son métier : "Les écrivains " - constate-t-elle " doivent ainsi, comme nous professeurs des universités, travailler à leur visibilité, s'ils veulent intégrer la République des lettres ${ }^{18}$. De même, Jean-Benoît Puech, qui dans une étude consacrée aux biographies fictionnelles postule une définition élargie du biographique contemporain, insiste en fait sur la nécessité de sortir du modèle strictement narratif de la construction d'une biographie d'écrivain : "Pour moi ", écrit-il, "le biographique désigne toutes les activités, discursives ou non, qui contribuent à la représentation de la personne et de la vie de l'auteur, ou plus précisément, qui transforment sa personne réelle en personnage. C'est un ensemble très vaste dont la biographie n'est qu'un des éléments, peut-être la synthèse discursive et iconique [je souligne] ${ }^{19}$. Par ailleurs, cette opinion semble être partagée par Viart lui-même lorsqu'il constate que " la dimension narrative en effet ne rend pas suffisamment compte du biographique ${ }^{20}$. Toutes ces voix, qui viennent en fait de l'intérieur de la « culture

\footnotetext{
17 Ibidem.

18 P. A. Boué, « Jean-Marie Gustave Le Clézio et Patrick Modiano dans le champ intellectuel européen ", op. cit.

19 J.-B. Puech, « Fiction biographique et biographie fictionnelle. L'auteur en représentation ", [dans :] F. Dion, R. Regard (dir.), Les nouvelles écritures biographiques, Lyon, ENS Éditions, 2013, p. 30.

20 D. Viart, " Dis-moi qui te hante. Paradoxes du biographique ", [dans :] Revue des sciences humaines, 2001, no 263, p. 11.
} 
de l'écrit ", semblent réclamer une reconnaissance de la visibilité comme un élément important de l'identité de l'écrivain. En même temps, la visibilité, comme l'a montré Nathalie Heinich dans une étude consacrée au phénomène contemporain de la célébrité, peut constituer un capital transmissible ${ }^{21}$. Or, un écrivain peut-il transmettre un héritage visuel ? Est-il capable de le faire par le biais des mots et de la langue?

\section{Contre la visibilité : le cas de Patrick Modiano}

Lors d'un débat organisé au Théâtre de l'Odéon en 1996 à l'occasion de l'exposition de seize portraits de Jacques Derrida, le philosophe déclarait : " II y a quelqu'un que j'admire à la télévision, si un jour j'y vais, il faudrait que je fasse comme lui, c'est Modiano. Voilà quelqu'un qui a réussi à transformer la scène publique et à la plier à un rythme qui est le sien [...] ${ }^{22}$. En effet, Patrick Modiano, dès la publication de son premier roman, La Place de l'étoile, adopte une attitude réservée par rapport aux médias, à l'encontre d'un Bernard-Henri Lévi ou d'un Michel Houellebecq considérés par les journalistes comme " des stratèges de la communication [qui] ont un fiévreux souci de leur image ${ }^{23}$. Ses difficultés d'élocution devant la caméra sont aujourd'hui légendaires et l'on sait que l'écrivain évite de s'exposer à la vue du public. II avoue, par exemple, à propos des séances de signature promotionnelles : "L'écriture est un métier où on est complètement déconnecté, toujours seul. Ce n'est pas un

${ }^{21} \mathrm{~N}$. Heinich, De la visibilité. Excellence et singularité en régime médiatique, Paris, Gallimard, 2012.

22 J. Derrida, "Portrait d'un philosophe : Jacques Derrida ", [dans :] Philosophie, philosophie, Université de Paris VIII, numéro hors-série, 1997. Cité d'après J. Meizoz, La littérature "en personne ". Scène médiatique et formes d'incarnation, Genève, Slatkine, 2007, p. 7.

23 J. Garcin, "Un Nobel anti-parisien : Le Clézio, l'ami public », [dans :] L'Obs [on-line]. Cité d'après P. A. Boué, " Jean-Marie Gustave Le Clézio et Patrick Modiano dans le champ intellectuel européen », op. cit. 
travail collectif comme celui des metteurs en scène de théâtre, par exemple [...] sauf, bien sûr, à donner des conférences au PEN Club ou à pratiquer des séances de signatures [...]. À mon époque, ça n'existait pas les signatures, alors je continue comme ça, il est normal de ne pas $m^{\prime} y$ voir ${ }^{24}$. Acceptant d'être photographié, l'auteur de Dora Bruder se laisse toutefois prendre sans retouches et corrections, indifférent aux impératifs esthétiques imposés par les médias visuels. Sur le portrait qui accompagne l'interview avec Modiano à l'occasion de la publication de son Horizon en 2010, le journaliste fournit l'information suivante, jugeant nécessaire d'expliquer pourquoi la photographie n'a pas été, comme à l'accoutumée, traitée : "Patrick Modiano chez lui à Paris. Sur les photos de face, faire attention à son œil droit, un vaisseau capillaire ayant explosé la veille de la prise de vue. Par contre Patrick Modiano n'a aucun problème pour être photographié avec son $œ i l$ rouge ${ }^{25}$. Ajoutons aussi que Modiano est un utilisateur sceptique des nouvelles technologies, il avoue, par exemple, se sentir mal à l'aise avec Internet qui, du fait d'une visualisation trop rapide à son sens des réponses sur l'écran, rend difficile le travail de l'imagination ${ }^{26}$.

L'écrivain est ainsi connu non seulement pour son œuvre, mais aussi (sinon surtout) pour sa façon d'être publique et s'il existe, en général, un consensus autour de son attitude, il est difficile de la considérer comme tout à fait non orchestrée. Henri Raczymow, dans l'essai déjà cité, affirme : "La timidité de Patrick Modiano avait en effet, aux beaux jours d'Apostrophes, tous les traits de

${ }^{24}$ M. Payot, D. Peras, " Patrick Modiano : "Je suis devenu comme un bruit de fond" ", [dans :] L'Express [on-line], 4 mars 2010, https://www.lex press.fr/culture/livre/patrick-modiano-je-suis-devenu-comme-un-bruit-defond_852718.html.

25 Ibidem.

${ }^{26}$ S. Bourneau, Patrick Modiano. Du bon usage d'Internet en littérature. Entretien par Sylvaine Bourneau, Paris, février 2010, https://www.youtube.com/ watch?v=NPoe5WzO3WA. 
l'archétype : elle surprenait au départ puis pouvait charmer [...] ou agacer. En réalité, Patrick Modiano jouait à merveille son personnage de bègue, mais c'est le metteur en scène Pivot qui le distribuait et le public qui l'attendait précisément dans ce rôle ${ }^{27}$. D'un côté, une telle attitude relève de la résistance manifestée par les gens de lettres contre la visibilité dont se nourrit la célébrité : " Le discrédit attaché à la visibilité dans le monde littéraire est si fort que peuvent même s'y produire des stratégies d'invisibilité ${ }^{28}$, explique Nathalie Heinich. Mais d'un autre côté, Modiano est justement (re)connu grâce à la consécration médiatique de son "désir de disparaître "29. Or, plus l'écrivain est devenu célèbre, plus ses efforts pour fuir le " spectacle » se sont accentués. L'interview organisée chez Gallimard à l'occasion de l'attribution du prix Nobel semble en apporter une nouvelle preuve - ce qui n'a pas manqué de susciter des voix critiques qui, commentant sa difficulté à formuler les phrases, lui ont conseillé de consulter un orthophoniste ${ }^{30}$. Modiano serait-il devenu victime de sa propre image d'écrivain invisible?

\section{Marie Modiano : un retour au « spectacle »}

Marie Modiano, née en 1978, est la seconde fille de Patrick Modiano et Dominique Zehrfuss, illustratrice, et la sœur de Zina Modiano, réalisatrice. Elle a grandi à Paris et est partie faire des études d'art dramatique à Londres. Après ses débuts de comédienne, elle a commencé à écrire et à chanter. Elle a signéà ce jour cinq albums et publié cinq œuvres littéraires : deux romans, deux recueils de

${ }^{27} \mathrm{H}$. Raczymow, La mort du grand écrivain. L'essai sur la fin de la littérature, op. cit., p. 146-147.

${ }^{28} \mathrm{~N}$. Heinich, De la visibilité. Excellence et singularité en régime médiatique, op. cit., p. 161.

${ }^{29}$ Cf. D. Rabaté, Désirs de disparaître. Une traversée du roman français contemporain, Québec, Tangence Éditeurs, 2016.

${ }^{30}$ P. A. Boué, "Jean-Marie Gustave Le Clézio et Patrick Modiano dans le champ intellectuel européen », op. cit. 
poésie et un ouvrage écrit en collaboration avec ses parents, sans pour autant prétendre se considérer comme un véritable écrivain : "Pour avoir grandi auprès d'un grand écrivain ", dit-elle modestement dans une interview, « il m'est difficile de me dire écrivain juste parce que j'ai publié quelques livres. Je suis plutôt une musicienne qui écrit ${ }^{31}$. Pourtant, il est peut-être difficile de ne pas écrire lorsqu'on reçoit l'écriture en héritage. Non seulement, dès son plus jeune âge, Marie a composé des poèmes, mais elle a pris aussi l'habitude de remplir des agendas de notes, de manière à noircir complètement les pages, comme si l'écriture était pour elle un geste quotidien, une manière de se trouver des repères, voire même " un besoin vital ": " noter permet de distinguer une journée d'une autre ", avoue-t-elle, " sinon elles finissent par se ressembler toutes ${ }^{32}$. En tant qu'écrivaine, Marie partage sans doute les plus grandes obsessions de son père. Dans Upsilon Scorpii (2013) la narratrice, jeune fille enfermée dans une ville imaginaire et luttant contre une mystérieuse maladie, part un jour à la recherche de son ami, Freddie, dont elle a partagé la vie et qui s'est esquivé de manière inexplicable. Le roman Lointain (2017) est construit autour de la disparition d'un jeune écrivain américain et renvoie à une histoire vécue, celle de la relation amoureuse de Marie adolescente avec l'écrivain Tristan Egolf qui s'est suicidé en 2005 et dont le premier roman, Le Seigneur des porcheries, plusieurs fois refusé dans les maisons d'édition américaines, a été publié en France grâce au soutien de Patrick Modiano et a reçu un bon accueil de la critique. Comme son père, Marie prétend écrire à la fois avec la disparition et contre elle. En commentant

31 M. Tran Huy, "Marie Modiano : "Je suis une musicienne qui écrit" ", [dans :] Madame Figaro, 2 mars 2018, http://madame.lefigaro.fr/celebrites/ marie-modiano-je-suis-une-musicienne-qui-ecrit-020318-147559.

32 M. Aïssaoui, " Nom : Modiano Prénom : Marie », [dans :] Figaro, 25 octobre 2013, http://premium.lefigaro.fr/mon-figaro/2013/10/25/1000120131025ARTFIG00453-nom-modiano-prenom-marie.php. 
la publication de son recueil poétique, Espérance mathématique (2012), Marie a dit ceci : "Écrire des poèmes est une manière de graver, d'imprimer pour toujours des instants, des lieux, ou des personnes qui sont voués à disparaître dans l'immense puits qu'est la mémoire ${ }^{33}$. Cependant, au-delà d'être simplement un sujet littéraire, la disparition est aussi une espèce de credo de toute la famille Modiano. Sur la quatrième de couverture du volume préparé par Marie en collaboration avec ses parents, Dominique Zehrfuss, sa mère, déclare : "Lorsqu'on se "fait tout petit", on peut disparaître, mais c'est peut-être pour mieux renaître dans un autre monde, un jardin d'Éden ${ }^{34}$.

Or, Marie a finalement préféré, en devenant actrice et chanteuse, la scène à l'écriture, et donc la visibilité à l'invisibilité. À l'encontre de son père, elle aime les concerts et le contact direct avec le public ${ }^{35}$, tout en se distanciant, cependant, du voyeurisme et de l'exhibitionnisme apportés par la culture visuelle : "Le déversement de l'intimité sur les réseaux sociaux me gêne plus qu'il ne m'agace ", avoue-t-elle, " comme si l'on avait besoin du regard des autres et de leur approbation pour exister $»^{36}$. Cette distanciation se fait ressentir aussi bien lors de ses concerts que dans ses romans. Elle chante d'une voix feutrée en s'accompagnant elle-même au piano ou en se faisant accompagner à la guitare par son mari, Peter von Poehl, pro-

33 G. Sbalchiero, "Marie Modiano: "Écrire est un besoin vital” ", [dans :] L'Express, 10 février 2012, https://www.lexpress.fr/culture/livre/mariemodiano-ecrire-est-un-besoin-vital_1080183.html.

${ }^{34}$ M. Modiano, P. Modiano, D. Zehrfuss, 28 paradis, 28 enfers, Paris, Gallimard, 2012. C'est un petit volume combinant les textes poétiques de Patrick et de Marie et les gouaches de Dominique, qui représentent 28 miniatures de paradis et $\mathbf{2 8}$ miniatures d'enfers rêvés par l'illustratrice à la manière de peintres japonais du XVIII siècle qui s'amusaient à présenter le monde " réduit à l'infiniment petit ».

35 D. Desreumaux, "Marie Modiano, "la scène est une récompense" ", [dans :] Hexagone, Revue Trimestrielle de la Chanson, (8 décembre 2014), hexagone.me/2014/12/marie-modiano/.

${ }^{36}$ M. Tran Huy, "Marie Modiano : "Je suis une musicienne qui écrit" ", op. cit. 
posant un mode discret d'être sur la scène : " À la voir, à l'entendre ", écrivit un journaliste, "Marie Modiano pourrait être l'un des personnages féminins qui traversent les romans de son père : taiseuse, timide, mystérieuse ${ }^{37}$. Il en est de même pour ses romans. Dans Upsilon Scorpii, la narratrice ressent un besoin permanent de se cacher. Dans Lointain, la narratrice, d'abord comédienne en tournée, ensuite chanteuse débutante obligée de plaire au public, vit à son tour l'exposition sur la scène comme une expérience déshumanisante :

Elle était installée devant son piano et ne distinguait pas grand-chose dans l'auditoire, à cause des projecteurs braqués sur elle. Les sièges paraissaient tous vides, elle n'aperçut que deux visages dans l'ombre, placés à chacune des extrémités d'une rangée. Elle sentait qu'on la fixait curieusement, comme une bête de foire, et cela la mit mal à l'aise. $(L, 129)$

Le titre du roman, Lointain, qui dans le vocabulaire théâtral signifie l'arrière de la scène, la partie du plateau la plus éloignée des spectateurs, traduit ainsi la volonté de la narratrice de rester à l'abri des regards des autres. Mais il correspond aussi à cette forme discrète de visibilité pratiquée par Marie Modiano elle-même, à son retour au "spectacle ", réalisé de manière non médiatique et de l'intérieur de la culture de l'écrit.

La ressemblance des manières d'être en public de Patrick Modiano et de sa fille, Marie, montre que la thèse à propos de la rupture de la filiation dans le nouveau contexte médiatique semble en fait être lancée du point de vue d'une littérature considérée comme autotélique. Or la réflexion sur la filiation non narrative, qu'il me semble tout à fait justifié de postuler aujourd'hui, loin de prétendre substituer celle-ci à son correspondant narratif, a tout de même toutes les chances de l'enrichir et de le compléter par la prise en considération aussi bien de l'image médiatique de l'écrivain que des possibilités de sa transmission. Comme le récit de filiation, et conformé-

37 M. Aïssaoui, « Nom : Modiano, Prénom : Marie », op. cit. 
ment au modèle qui a été présenté par Dominique Viart lors de sa communication ${ }^{38}$, la filiation non narrative réussit également à proposer, nous l'avons vu avec l'exemple de Marie Modiano, une poétique (le cas échéant, celle de la visibilité lorsque Marie se présente comme incarnation des personnages de l'univers romanesque de son père) et une éthique de la restitution (I'univers médiatique que se réapproprie la fille chanteuse au nom du père-écrivain). C'est donc finalement Marie qui non seulement transforme le " désir de disparaître " de son père en un " capital " transmissible, mais - à travers sa présence sur la scène - renforce, voire même affirme la " posture littéraire " $^{39}$ de Patrick Modiano lui-même. La filiation non narrative, qui trouve ainsi de nouveaux lieux, non strictement littéraires, de production et de diffusion (tels confidences ou entretiens médiatisés) et qui peut être en fin de compte considérée comme une réception médiatique de la relation qui lie un écrivain à un parent, permet au récit de filiation de s'intégrer à la culture visuelle.

Date de réception de l'article : 20.05.2019. Date d'acceptation de l'article : 14.06.2019.

38 Je me réfère à la communication introductive de Dominique Viart lors du colloque "Le récit de filiation : dimensions et extensions ", Gdańsk, 14-15 mai 2019.

${ }^{39}$ Le concept de " posture " renvoie aux conduites non-verbales de l'auteur dans le champ littéraire en relation à son " ethos discursif ", son agir linguistique : l'activité littéraire est ainsi considérée conjointement comme conduite et comme discours. Cf. J. Meizoz, La littérature "en personne ". Scène médiatique et formes d'incarnation, op. cit., p. 13. 


\section{bibliographie}

Aïssaoui M., " Nom : Modiano Prénom : Marie ", [dans :] Le Figaro, 25 octobre 2013, http://premium.lefigaro.fr/mon-figaro/2013/10/25/1000120131025ARTFIG00453-nom-modiano-prenom-marie.php.

Bonnet J.-C., Naissance du Panthéon. Essai sur le culte des grands hommes, Paris, Fayard 1998.

Boué P. A., "Jean-Marie Gustave Le Clézio et Patrick Modiano dans le champ intellectuel européen ", [dans :] Carnets [Online], Deuxième série, 2017, no 9, http://journals.openedition.org/carnets/2052

Bourneau S., Patrick Modiano. Du bon usage d'Internet en littérature. Entretien par Sylvaine Bourneau, Paris, février 2010, https://www.youtube.com/ watch?v=NPoe5WzO3WA.

Compagnon A., La Troisième République des lettres. De Flaubert à Proust, Paris, Seuil, 1983.

Demanze L., Encres orphelines. Pierre Bergounioux, Gérard Macé, Pierre Michon, Paris, José Corti, 2008.

Desreumaux D., " Marie Modiano, "la scène est une récompense" ", [dans :] Hexagone, Revue Trimestrielle de la Chanson, 8 décembre 2014, www. hexagone.me/2014/12/marie-modiano/.

Heinich N., De la visibilité. Excellence et singularité en régime médiatique, Paris, Gallimard, 2012.

Kaufmann V., Les dernières nouvelles du spectacle. Ce que les médias font à la littérature, Paris, Seuil, 2017.

Meizoz J., La littérature " en personne ». Scène médiatique et formes d'incarnation, Genève, Slatkine, 2007.

Modiano M., Espérance mathématique, Paris, Gallimard, 2012.

Modiano M., Upsilon Scorpii, Paris, Gallimard, 2013.

Modiano M., Lointain, Paris, Gallimard, 2017.

Modiano M., Pauvre Chanson, Paris, Gallimard, 2018.

Modiano M., Modiano P., Zehrfuss D., 28 paradis, 28 enfers, Paris, Gallimard, 2012.

Nora O., "La visite au grand écrivain ", [dans :] P. Nora (dir.), Les lieux de mémoire, Paris, Gallimard, 1986, t. 2 ("La Nation »), vol. 3.

Payot M., Peras D., " Patrick Modiano : "Je suis devenu comme un bruit de fond" ", [dans :] L'Express [on-line], 4 mars 2010, https://www.lexpress.fr/ culture/livre/patrick-modiano-je-suis-devenu-comme-un-bruit-de-fond_ 852718.html.

Prosowska M., Le récit de filiation dans la littérature contemporaine en France et en Pologne, thèse de doctorat non publiée, sous la direction de J. Lis, Poznań, Université Adam Mickiewicz, 2007.

Puech J.-B., " Fiction biographique et biographie fictionnelle. L'auteur en représentation ", [dans :] F. Dion, R. Regard (dir.), Les nouvelles écritures biographiques, Lyon, ENS Éditions, 2013.

Rabaté D., Désirs de disparaître. Une traversée du roman français contemporain, Québec, Tangence Éditeurs, 2016.

Raczymow H., La mort du grand écrivain. L'essai sur la fin de la littérature, Paris, Stock, 1996 (ebook). 
Sbalchiero G., "Marie Modiano: "Écrire est un besoin vital" ", [dans :] L'Express, 10 février 2012, https://www.lexpress.fr/culture/livre/mariemodiano-ecrire-est-un-besoin-vital_1080183.html.

Tran Huy M., "Marie Modiano : "Je suis une musicienne qui écrit" ", [dans :] Madame Figaro, 2 mars 2018, http://madame.lefigaro.fr/celebrites/mariemodiano-je-suis-une-musicienne-qui-ecrit-020318-147559.

Viart D., " Dis-moi qui te hante. Paradoxes du biographique ", [dans :] Revue des sciences humaines, 2001, n०263.

Viart D., "Filiations littéraires ", [dans :] J. Baetens, D. Viart (dir), Écritures contemporaines 2. États du roman contemporain, Actes du colloque de Calaceite, 6-13 juillet 1996, Paris-Caen, Lettres Modernes Minard, 1999.

\section{abstract}

The author in situation of the "spectacle": towards a non-narrative filiation

The paper discuss the concept of "filiation narratives", proposed in 1999 by the French theorist Dominique Viart, from the point of view of the "spectacularization of the author" (V. Kaufmann) in the contemporary visual culture. On the one hand, in visual culture, the media visibility of the author seems much important that his work. On the other hand, the strictly narrative understanding of the filiation is no longer possible. The example of the non-narrative filiation which connect a French writer Patrick Modiano and his daughter, singer et writer Marie Modiano, is proposed to show the resistance of the literature against the abuses of the media's influence to the literary culture.

\section{keywords}

filiation narratives, non-narrative filiation, spectacle, author, visibility, invisibility

\section{mots-clés}

récit de filiation, filiation non narrative, spectacle, auteur, visibilité, invisibilité 


\section{katarzyna thiel-jańczuk}

Katarzyna Thiel-Jańczuk est docteur en lettres modernes françaises et maître de conférences à l'Université Adam Mickiewicz à Poznań. Elle s'intéresse aux fictions biographiques contemporaines ainsi qu'à la problématique de la filiation dans l'écriture française contemporaine. Elle prépare un livre consacré à la problématique du portrait littéraire et de l'écrivain comme représentation. Elle a publié les travaux consacrés à l'œuvre de Patrick Modiano, Annie Ernaux, Pierre Michon, Pascal Quignard, Roland Barthes. Elle est aussi traductrice en polonais des œuvres, entre autres, de Michel de Certeau et Claude Lévi-Strauss.

ORCID : 0000-0002-9418-5231 